\title{
Prevalência de Infecção Nosocomial em Unidades de Terapia Intensiva do Rio Grande do Sul*
}

\author{
The Prevalence of nosocomial infection in Intensive \\ Care Units in the State of Rio Grande do Sul
}

\author{
Thiago Lisboa', Mario Faria', Jorge A. Hoher',8, Luis A.A. Borges ${ }^{3}$, Jussara Gómez ${ }^{4}$, \\ Luciele Schifelbain ${ }^{5}$, Fernando S. Dias ${ }^{6}$, João Lisboa ${ }^{7}$, Gilberto Friedman ${ }^{1,8}$
}

\section{RESUMO}

JUSTIFICATIVA E OBJETIVOS: Determinar a prevalência de infecções adquiridas em UTI e os fatores de risco para estas infecções, identificar os organismos infectantes mais prevalentes, avaliar a relação entre infecção adquirida na UTI e mortalidade.

MÉTODO: Estudo de prevalência de um dia. Participaram do estudo 16 UTI do estado do Rio Grande do Sul, excluindo unidades coronarianas ou pediátricas. Todos os pacientes com idade maior que 12 anos, ocupando um leito de UTI por um período de $24 \mathrm{~h}$, foram incluídos. As 16 UTI coletaram dados de 174 pacientes. Principais desfechos: taxas de infecção adquirida na UTI, padrões de resistência dos patógenos isolados e fatores potenciais de risco para infecção adquirida na UTI e mortalidade.

1. Serviço de Medicina Intensiva do Hospital de Clínicas de Porto Alegre.

2. UTI do Hospital Cristo Redentor.

3. UTI do Hospital Nossa Senhora da Conceição.

4. UTI do Hospital São Vicente de Paula.

5. UTI Hospital de Caridade Astrogildo de Azevedo.

6. UTI do Hospital Universitário São Lucas da PUCRS.

7. UTI do Hospital de Caridade de ljuí.

8TI Central do Complexo Hospitalar da Santa Casa de Porto Alegre.

*Recebido da Unidade de Terapia Intensiva de Porto Alegre, RS

Apresentado em 10 de dezembro de 2007

Aceito para publicação em 18.12.07

Endereço para correspondência:

Dr. Gilberto Friedman

Universidade Federal do Rio Grande do Sul

Hospital de Clínicas de Porto Alegre

Largo Eduardo Zaccaro Faraco - Rua Ramiro Barcelos, 2350

90035-903 - Porto Alegre, RS

Fone: (51) 2101-8634; Fax (51) 2101-8526

E-mail: gfriedman@hcpa.ufrgs.br. gfried@portoweb.com.br

(C)Associação de Medicina Intensiva Brasileira, 2007
RESULTADOS: Um total de 122 pacientes (71\%) estava infectado, e 51 (29\%) adquiriram infecção na UTI. Pneumonia $(58,2 \%)$, infecção do trato respiratório inferior $(22,9 \%)$, infecção do trato urinário $(18 \%)$ foram os tipos mais freqüentes de infecção. Os microorganismos mais relatados foram stafilococos aureus (42\% [64\% resistentes a oxacilina]) e pseudomonas aeruginosa (31\%). Seis fatores de risco foram identificados para infecção adquirida na UTI: cateter urinário, acesso vascular central, intubação traqueal por tempo prolongado ( $>4$ dias), doença crônica, trauma e internação prolongada na UTI (> 30 dias). Os fatores de risco associados à morte foram idade, APACHE II, falência orgânica e prótese em via aérea com ou sem ventilação mecânica.

CONCLUSÕES: A infecção adquirida na UTI é comum e freqüentemente associada a isolados de microorganismos resistentes. Este estudo, apesar de sua abrangência regional, serve de referência epidemiológica para ajudar a programar políticas de controle de infecção.

Unitermos: epidemiologia, infecção, mortalidade, prevalência, UTI

\section{SUMMARY}

BACKGROUND AND OBJECTIVES: To determine the prevalence of intensive care unit (ICU)-acquired infections and the risk factors for these infections, identify the predominant infecting organisms, and evaluate the relationship between ICU-acquired infection and mortality.

METHODS: A 1-day point prevalence study. Sixteen ICU of the State of Rio Grande do Sul-Brazil, excluding coronary care and pediatric units. All patients $<12$ yrs occupying an ICU bed over a 24-hour period. The 16 ICU provided 174 case reports. Main outcomes: rates of ICU-acquired infection, resistance patterns of microbiological isolates, and potential risks factors for ICUacquired infection and death. 
RESULTS: A total of 122 patients $(71 \%)$ was infected and $51(29 \%)$ had ICU-acquired infection. Pneumonia $(58.2 \%)$, lower tract respiratory infection (22.9\%), urinary tract infection (18\%) were the most frequents types of ICU infection. Most frequently microorganisms reported were staphylococcus aureus (42\% [64\% resistant to oxacilin]) and pseudomonas aeruginosa (31\%). Six risk factors for ICU acquired infection were identified: urinary catheterization, central vascular line, tracheal intubation for prolonged time ( $>4$ days), chronic disease and increased length of ICU stay ( $>30$ days). The risks factors associated with death were age, APA$\mathrm{CHE}$ II, organ dysfunction, and tracheal intubation with or without mechanical ventilation.

CONCLUSIONS: ICU-acquired infection is common and often associated with microbiological isolates of resistant organisms. This study may serve as an epidemiological reference to help the discussion of regional infection control policies.

Key Words: epidemiology, ICU, infection, mortality, prevalence

\section{INTRODUÇÃO}

As doenças infecciosas estão entre as mais prevalentes nas Unidades de Terapia Intensiva (UTI), sendo importante fator implicado no desfecho desfavorável dos pacientes criticamente enfermos ${ }^{1,2}$. Quadros infecciosos em UTI estão associados com maior tempo de internação, maiores morbidade e mortalidade, além do alto custo $^{3}$. Dados de um estudo transversal europeu sobre infecção (EPIC) mostram uma prevalência de $45 \%$ no dia da avaliação ${ }^{1}$. Outro estudo que incluiu o Canadá e Israel além de seis países europeus mostrou que $20,6 \%$ dos pacientes admitidos em UTI estavam infectados ${ }^{4}$. A mortalidade nos pacientes infectados foi quatro vezes maior que no grupo sem infecção. Angus e col. analisaram mais de 6 milhões de registros de altas hospitalares nos EUA e estimaram 751.000 casos de sepse grave anualmente e com uma mortalidade de $28,6 \%^{3}$. Outros estudos epidemiológicos na Europa e Oceania mostraram que pacientes com infecções graves apresentam mortalidade hospitalar que variam de $27 \%$ a $55 \%^{5-7}$. No México, outro estudo transversal em 254 UTI, observou que 58\% dos pacientes estavam infectados e entre estes $22 \%$ faleceram ${ }^{8}$.

Infelizmente, dados brasileiros confiáveis a respeito da prevalência de infecção em UTI são escassos e, conseqüentemente, a avaliação do seu impacto na morbimortalidade e dos custos associados é limitada.
No Brasil, alguns estudos avaliaram o impacto das infecções em ambiente de terapia intensiva. Toufen Jr e col. estudaram a prevalência de infecção nas UTI de um Hospital Universitário e encontraram uma taxa alta de infecção e predominaram bactérias resistentes ${ }^{9}$. Estudos brasileiros recentes que enfocaram os aspectos epidemiológicos da síndrome séptica e não exatamente das infecções em ambiente de UTI mostram que o pulmão foi o foco de infecção mais importante e os bacilos Gram-negativos e cocos Gram-positivos foram principais agentes responsáveis ${ }^{10,11}$. A taxa de mortalidade nestes estudos variou de $34,7 \%$ até $46,6 \%$ e atingindo $65 \%$ na presença de choque séptico.

Entretanto, o Brasil possui uma população extremamente heterogênea no que diz respeito a diferentes realidades regionais bem como diferentes condições de acesso aos serviços de saúde, tendo estes fatores implicação no desenvolvimento de políticas que visem um rastreamento e controle de infecção adequado.

Não existe no Brasil, com exceção de esforços localizados e relacionados a protocolos de pesquisa, um sistema para rastreamento e controle de infecção bem organizado que permita uma avaliação adequada das infecções nosocomiais em pacientes criticamente enfermos que permita uma estimativa adequada da importância deste problema, permitindo a partir destes dados a avaliação de fatores de risco, morbimortalidade e micro-organismos mais comumente envolvidos, permitindo que se detectem variabilidades regionais $e$ a melhor estratégia no controle deste problema.

O objetivo deste estudo foi avaliar a prevalência de infecção nosocomial, bem como identificar os fatores de risco associados, as áreas mais freqüentemente acometidas e os germes mais freqüentemente envolvidos em UTI localizadas no estado do Rio Grande do Sul.

\section{MÉTODO}

Foi enviado aos diretores de 16 UTI no estado do Rio Grande do Sul um convite para participarem de um estudo transversal de prevalência de um dia. Foram excluídas unidades pediátricas e coronarianas. Um responsável pela coleta dos dados foi nomeado por unidade. A população foi definida como sendo todos os pacientes internados ao longo de um período de $24 \mathrm{~h}$ iniciado a partir de meia noite. Pacientes com menos de 12 anos foram excluídos do estudo.

Para cada paciente foram coletados, através de formulário padrão e enviado as unidades por correio eletrônico, dados demográficos, doenças de base, estado clí- 
nico na admissão, paciente clínico/cirúrgico, disfunção orgânica, escore Acute Physiology and Chronic Health Evaluation II (APACHE II) ${ }^{12}$, antibióticos e estado da infecção. Os fatores de risco coletados incluíram presença de sonda vesical, tubo traqueal, traqueostomia, ventilação mecânica, drenos, hemodiálise ou diálise peritoneal, cateter venoso central, nutrição parenteral, administração de imunossupressores ou profilaxia de úlcera de estresse.

A presença ou não de infecção foi definida de acordo com a equipe assistente, sendo classificada em comunitária, nosocomial ou nosocomial adquirida na UTI. Dados microbiológicos foram coletados sempre que disponíveis e foram buscados ativamente os dados que tivessem sido liberados ate uma semana após a data do estudo. O desfecho dos pacientes foi avaliado como alta ou óbito em até seis semanas após a data de estudo.

\section{Análise Estatística}

Uma análise descritiva foi realizada, com os valores sendo expressos como média \pm desvio-padrão (variáveis contínuas) ou como percentagem do grupo (variáveis categóricas). Todas as comparações foram feitas com um teste- $t$ para grupos não-pareados. As variáveis contínuas foram comparadas usando o teste $t$ de Student para variáveis com distribuição normal e o teste de Wilcoxon para variáveis com distribuição não- normal. $\mathrm{O}$ teste $\mathrm{X}^{2}$ foi utilizado para comparar variáveis categóricas.

Foi realizada uma regressão logística utilizando a razão de chances - $(\mathrm{RC})$ - para avaliar os efeitos de variáveis independentes como fatores de risco para a presença ou ausência de infecção. Um modelo de análise multivariada foi utilizado para avaliar o risco de infecção e o risco de morte e identificar fatores de risco independentes. Foi considerado significativo quando $p<0,05$.

\section{RESULTADOS}

Dezesseis UTI foram incluídas no estudo, sendo avaliados 174 pacientes distribuídos conforme a tabela 1 . Quatro UTI eram localizadas em hospitais universitários, nove em hospitais afiliados e três em hospitais comunitários. Dez UTI eram mistas (médico-cirúrgica) e as demais unidades eram especializadas em Trauma, Pneumologia, Neurologia, Oncologia e Transplantes de Órgãos. Onze UTI localizavam-se em Porto Alegre, das quais foram provenientes $75,7 \%$ dos pacientes.

Dos 174 pacientes estudados, 97 (56\%) eram homens, e a idade média foi de $56 \pm 18$ anos, com mediana de 60 anos. O tempo médio de permanência na UTI foi de 12,5 $\pm 22,5$ dias (Tabela 2). O APACHE II médio da amostra foi $18 \pm 9$ e foi calculado em 101 pacientes (58\%); na figura 1 é mostrada a sua distribuição.

A maioria dos pacientes incluía casos clínicos $(55,5 \%)$

Tabela 1 - Prevalência de Infecção por Centro

\begin{tabular}{|c|c|c|c|c|c|c|c|}
\hline Hospital & Tipo de UTI & $\mathrm{N}^{\circ}$ & $\%$ & Prevalêr & de Infecçã & $(\%) *$ & Taxa de Mortalidade na UTI (\%) \\
\hline & & & & Comunitária & Hospitalar & UTI & \\
\hline$A^{1}$ & Médico-cirúrgica & 22 & 12,6 & $11(50)$ & $5(23)$ & $3(14)$ & $10(48)$ \\
\hline $\mathrm{B}^{2}$ & Médico-cirúrgica & 20 & 11,5 & $6(30)$ & $12(60)$ & $8(40)$ & $12(60)$ \\
\hline $\mathrm{C}^{1}$ & Médico-cirúrgica & 4 & 2,3 & $3(75)$ & $1(25)$ & $1(25)$ & $1(25)$ \\
\hline$D^{2}$ & Trauma & 22 & 12,6 & 0 & $3(14)$ & $13(59)$ & $9(45)$ \\
\hline$E^{1}$ & Médico-cirúrgica & 13 & 7,5 & $2(15)$ & $3(23)$ & $5(38)$ & $4(31)$ \\
\hline $\mathrm{F}^{2}$ & Médico-cirúrgica & 14 & 8 & $5(36)$ & $2(14)$ & $5(36)$ & $6(43)$ \\
\hline $\mathrm{G}^{2}$ & Transplante de órgãos & 2 & 1,1 & 0 & 0 & $1(50)$ & $1(50)$ \\
\hline $\mathrm{H}^{2}$ & Oncologia & 8 & 4,6 & $2(25)$ & $2(25)$ & 0 & $1(14)$ \\
\hline I & Neurologia & 9 & 5,2 & 0 & 0 & $2(22)$ & $2(22)$ \\
\hline $\mathrm{J}^{2}$ & Pneumologia & 11 & 6,3 & $2(18)$ & $3(27)$ & $5(45)$ & $3(27)$ \\
\hline $\mathrm{K}^{2}$ & Trauma & 7 & 4 & $4(57)$ & $3(43)$ & $2(29)$ & - \\
\hline $\mathrm{L}^{3}$ & Médico-cirúrgica & 13 & 7,5 & $3(23)$ & $1(8)$ & $2(15)$ & $6(46)$ \\
\hline$M^{2}$ & Médico-cirúrgica & 3 & 1,7 & $1(33)$ & 0 & $1(33)$ & $1(33)$ \\
\hline $\mathrm{N}^{3}$ & Médico-cirúrgica & 9 & 5,2 & $3(33)$ & 0 & 0 & - \\
\hline $\mathrm{O}^{2}$ & Médico-cirúrgica & 3 & 1,7 & $2(67)$ & 0 & 0 & $1(33)$ \\
\hline $\mathrm{P}^{1}$ & Médico-cirúrgica & 14 & 8 & $3(21)$ & 0 & $4(29)$ & - \\
\hline Total & & 174 & 100 & $46(26)$ & $36(21)$ & $51(29)$ & 47 (45) \# \\
\hline
\end{tabular}

${ }^{*}$ O tipo de infecção foi informado para 113 dos 122 pacientes infectados. O percentual pode ser superior a $100 \%$ pois alguns pacientes apresentaram mais de um tipo de infecção.

\# O seguimento foi informado pra 104 pacientes, assim a taxa de mortalidade de alguns centros pode não ser a real.

${ }^{1}$ Hospital universitário, ${ }^{2} \mathrm{Hospital}$ afiliado, ${ }^{3} \mathrm{Hospital}$ comunitário 
e dentre os pacientes cirúrgicos a maior parte era de cirurgia de urgência (56\%). Vinte e oito pacientes (16\%) eram traumatizados e 69 (40\%) tinham alguma falência orgânica. A falência orgânica mais freqüente foi a pulmonar $(41,4 \%)$. Os principais dados clínicos e demográficos são apresentados na tabela 2 .

Tabela 2 - Dados Demográficos

\begin{tabular}{lc}
\hline Idade (anos) & $56 \pm 1$ \\
Sexo masculino (N e \%) & $97(56 \%)$ \\
Dias de internação pré-UTI & $6,8 \pm 12$ \\
Dias de internação na UTI & $12,5 \pm 22,5$ \\
APACHE II & $18 \pm 9$ \\
Óbito * & $47(45,2 \%)$ \\
Tipos de Internação (\%) & \\
Clínica & $55,5 \%$ \\
Cirúrgica & $31,2 \%$ \\
Mista & 13,3 \\
Cirurgias (N) & \\
Eletiva & 20 \\
Urgência & 26 \\
Falência orgânica & 69 \\
Pulmonar & 41,4 \\
Renal & 22 \\
Cardiovascular & 14 \\
Neurológica & 8 \\
Hematológica & 8 \\
Hepática & 7,5 \\
Trauma & 16 \\
Infecção & 70 \\
Infecção adquirida na UTI & 29 \\
\hline
\end{tabular}

*O seguimento foi informado para 104 dos 174 pacientes arrolados no estudo.

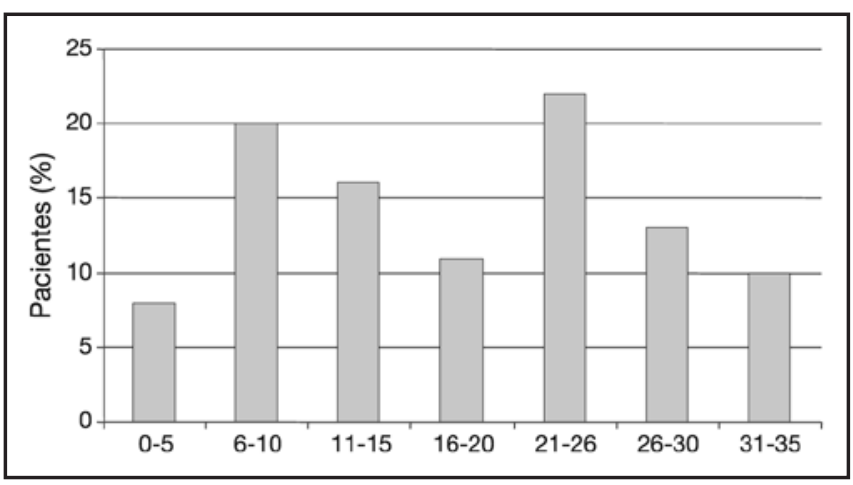

Figura 1 - Escore APACHE II para os 101 Pacientes que Tiveram seu Valor Calculado

\section{Prevalência de Infecção}

Um total de 122 pacientes (71\%) apresentava infecção no dia do estudo. Destas infecções a prevalência de infecções adquiridas na UTI foi de $29 \%$, pouco maior que as adquiridas na comunidade e que as nosoco- miais fora da UTI (Tabela 1).

A tabela 3 demonstra a prevalência de potenciais fatores de risco associados à infecção, relacionados às intervenções diagnósticas e terapêuticas. No dia do estudo, a intervenção mais freqüente foi a presença de sonda vesical $(72,5 \%)$, sendo importantes ainda a presença de cateter venoso (72\%), intubação e ventilação mecânica (57\%) e cateter para monitorização de pressão venosa central (50\%). Houve uma baixa prevalência de pacientes com síndrome da imunodeficiência adquirida (SIDA) na amostra (6\%), bem como de pacientes neutropênicos (3\%). A maioria dos pacientes utilizava alguma forma de profilaxia contra úlcera de estresse (62\%), a maioria com uso de bloqueador $\mathrm{H}_{2}$ (44\%). Apenas 19 pacientes nesta amostra faziam uso de corticóide no dia do estudo (11\%).

Tabela 3 - Potenciais Fatores de Risco para Infecção Nosocomial

\begin{tabular}{lcc}
\hline Intervenções & $\begin{array}{c}\text { Número de } \\
\text { Pacientes }\end{array}$ & $\%$ \\
\hline Cateter venoso & 125 & 72 \\
Cateter arterial & 18 & 10 \\
Cateter pulmonar & 9 & 5 \\
Cateter urinário & 126 & 72,5 \\
Pressão venosa central & 87 & 50 \\
Pressão intracraniana & 7 & 4 \\
Hemodiálise & 11 & 6 \\
Diálise peritoneal & 2 & 1 \\
Intubação traqueal & 99 & 57 \\
Ventilação mecânica & 99 & 57 \\
Traqueostomia & 33 & 19 \\
Nutrição parenteral periférica & 167 & 96 \\
Nutrição parenteral central & 9 & 5 \\
SIDA & 11 & 6 \\
Neutropenia & 5 & 3 \\
Terapia imunossupressiva & 6 & 3,5 \\
Corticóide em altas doses & 19 & 11 \\
Antibiótico profilático & 23 & 13 \\
Sedação & 65 & 37 \\
Profilaxia de úlcera & 108 & 62 \\
Bloqueador ${ }_{2}$ & 76 & 44 \\
Omeprazol & 32 & 18,5 \\
\hline
\end{tabular}

A área mais freqüentemente acometida pelo quadro infeccioso foi o pulmão em quase $60 \%$ dos casos, seguido por trato respiratório inferior, trato urinário (17\%) e sepse sem foco definido (12\%) (Tabela 5).

Os agentes microbiológicos foram identificados em apenas 64 pacientes (52\% dos pacientes infectados) e os germes mais freqüentemente isolados foram a: Stafilococos aureus isolado em $43 \%$ dos casos, sendo que destes $63 \%$ eram cepas resistentes a meticilina 
(MRSA); b: Pseudomonas aeruginosa isolada em $31 \%$ dos casos, sendo $6 \%$ das cepas multi-resistentes, c: Acinetobacter (22\%), d: Klebsiella (17\%); e: Escherichia Coli (15\%) (Figura 2).

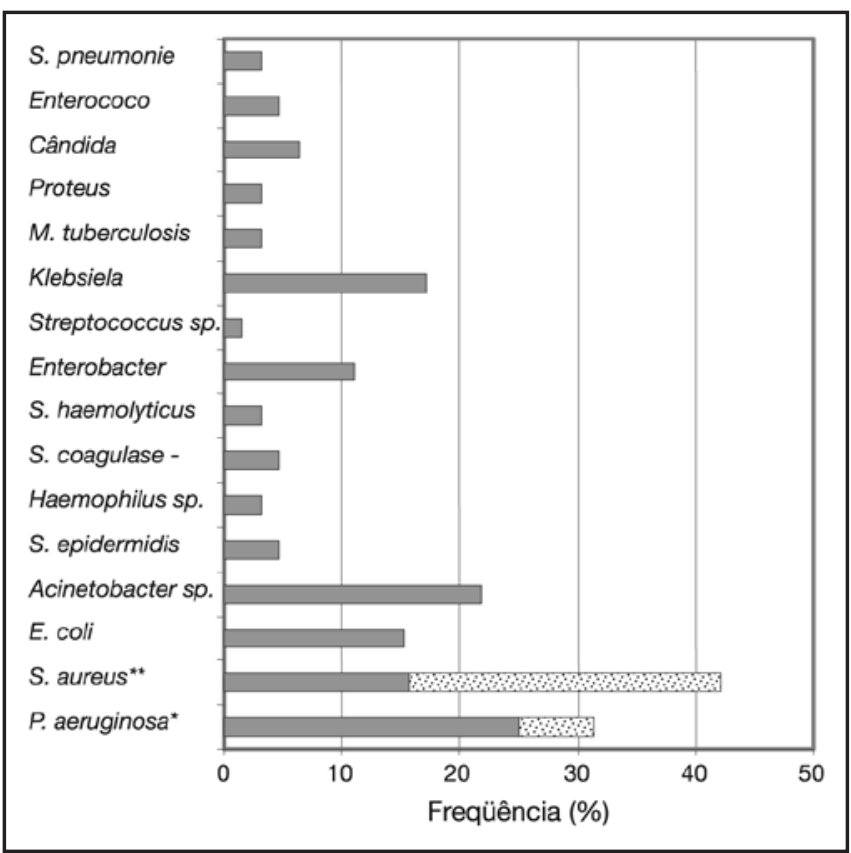

Figura 2 - Germes Isolados

- : dos 122 pacientes infectados, apenas 64 tiveram germes isolados.

: área pontilhada equivale às cepas resistentes a cefalosporinas.

** área pontilhada equivale às cepas resistentes a oxacilina.

\section{Fatores de Risco para Infecção Adquirida na UTI}

Entre os 122 pacientes infectados foi informado o tipo de infecção para 113. Pela análise univariada foram identificadas 10 variáveis associadas a risco de infecção adquirida na UTI. A presença de cateteres venoso central e urinário aparece como os principais fatores associados à infecção adquirida na UTI e com probabilidade oito vezes maior. Os fatores de risco usualmente implicados na ventilação mecânica prolongada, como insuficiência respiratória, disfunção neurológica e presença de traqueostomia foram significativamente associados à infecção em UTI. Da mesma forma, a ventilação mecânica per se afetou o risco. Os pacientes que apresentavam doenças crônicas (p. ex.: diabete melito, cardiopatia isquêmica) tinham quase a probabilidade de sete vezes maior de risco de infecção. Contudo, os pacientes traumatizados apresentaram um risco similar. O tempo de permanência em UTI foi um fator de risco importante para o desenvolvimento de infecção adquirida na UTI. Pacientes que permaneceram entre 7 e 30 dias na UTI tiveram uma chance associada significativamente maior para o desenvolvimento de infecção adquirida na UTI (razão de chances, $R C=3,25$, intervalo de confiança (IC) $95 \%(1,57-6,76), p=0,002)$. A permanência maior que por 30 dias esteve associada com chance ainda maior de infecção nosocomial adquirida em UTI (RC 6,19; IC 95\% [3,08 - 12,4], p = 0,001). Todos os pacientes que permaneceram por mais de trinta dias na UTI adquiriram infecção (Figura 3).

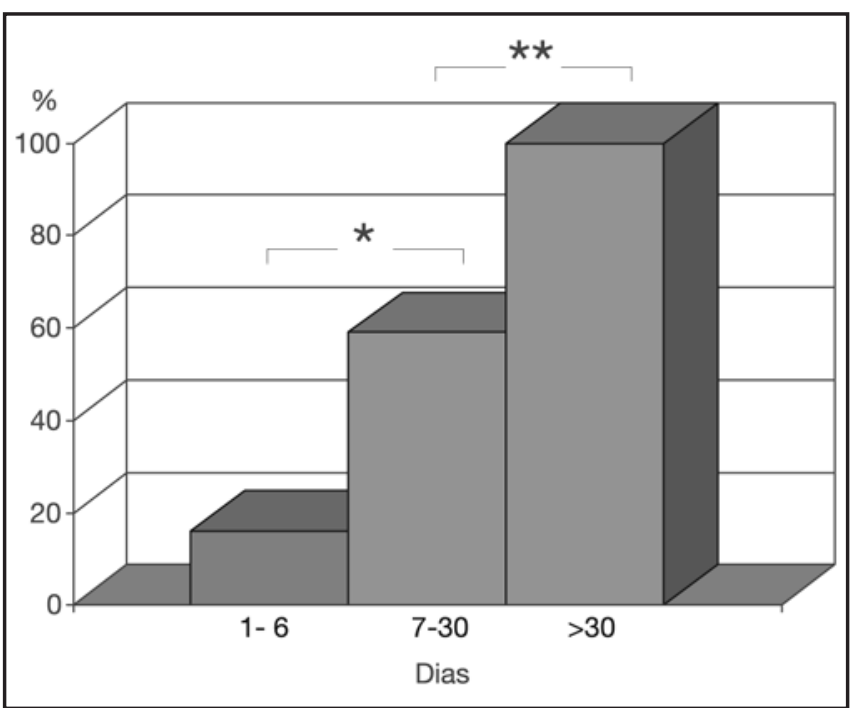

Figura 3 - Associação entre o Tempo de Internação e Infecção Adquirida na UTI.

* $p<0,05$ versus $1-6$ dias

${ }^{* *} p<0,05$ versus $7-30$ dias.

Uma análise de regressão logística foi realizada pra controlar os efeitos das variáveis de confusão. Todas as variáveis com associação significativa entraram no modelo. Os fatores de risco, associados com a necessidade de prótese em via aérea, foram agrupados (traqueostomia, intubação, ventilação mecânica). $\mathrm{Na}$ análise final, seis variáveis permaneceram como fatores associados de modo independente com a presença de infecção: cateterização urinária, acesso vascular central, prótese em via aérea por tempo prolongado, doença crônica, trauma e tempo prolongado de hospitalização na UTI (Tabela 4).

Tabela 4 - Fatores de Risco para Infecção Adquirida na UTI

\begin{tabular}{lccc}
\hline Fatores de Risco & $\mathrm{RC}$ & $\mathrm{IC} 95 \%$ & Valor de $\mathrm{p}$ \\
\hline Cateterização urinária & 8,63 & $1,40-8,24$ & 0,003 \\
Acesso vascular central & 8,62 & $1,36-2,75$ & 0,003 \\
Via aérea por tempo prolonga- & 8,6 & $1,39-2,74$ & 0,003 \\
do (> 4 dias) & & & \\
Doença crônica & 6,9 & $1,84-3,32$ & 0,03 \\
Trauma & 6,7 & $1,33-3,24$ & 0,01 \\
$>$ 30 dias de internação na UTI & 6,19 & $3,08-12,14$ & 0,001 \\
\hline
\end{tabular}

RC: razão de chances.

IC 95\%: intervalo de confiança de $95 \%$. 
Tabela 5 - Infecção e Locais Acometidos

\begin{tabular}{lc}
\hline & $\mathrm{N}^{\circ}(\%)$ \\
\hline Pneumonia & $71(58,2)$ \\
Trato respiratório inferior (não pneumonia) & $28(22,9)$ \\
Trato urinário & $22(18)$ \\
Sepse clínica sem foco & $16(13,1)$ \\
Bacteremia & $13(10,6)$ \\
Trato gastrintestinal & $8(6,6)$ \\
Ferida & $7(5,7)$ \\
Pele e tecidos moles & $6(4,9)$ \\
Sistema nervoso & $6(4,9)$ \\
Orelha, nariz e garganta & $3(2,5)$ \\
Cardiovascular & $2(1,6)$ \\
\hline
\end{tabular}

O APACHE II não foi diferente entre os pacientes que adquiriram infecções comunitárias, hospitalares e na UTI $(18 \pm 9$ versus $18 \pm 9$ versus $21 \pm 9 ; p=0,34)$ e não foi incluído no modelo. Entretanto, os valores de APACHE II foram superiores para os pacientes infectados que nos não infectados $(21 \pm 8$ versus $11 \pm 7$, p $<0,001)$.

\section{Fatores de Risco para Morte}

O seguimento foi avaliado nas seis semanas que se sucederam ao dia do estudo. Foram obtidos dados de 104 pacientes (59\% dos pacientes) e a mortalidade global foi de $45 \%$. Na análise univariada, a qual considerou a relação entre mortalidade e várias variáveis de risco os seguintes fatores de risco aumentaram as chances de óbito: idade, APACHE II, ventilação mecânica, falência orgânica múltipla, cateterização urinária e via aérea. Verificou-se uma tendência a maior mortalidade no grupo com infecção adquirida na UTI (48\%) quando comparado com pacientes sem infecção adquirida na UTI (41\%), porem essa diferença não foi significativa. $\mathrm{Na}$ análise de regressão logística, quatro variáveis foram independentemente associadas ao óbito: idade maior que 60 anos, APACHE II maior que 21, a falência orgânica múltipla e via aérea. Os pacientes não sobreviventes eram mais velhos (58 \pm 14 anos versus $48 \pm 20$ anos, $p=0,003$ ), apresentaram APACHE II mais elevado $(21 \pm 8$ versus $16 \pm 10, p=0,044)$, desenvolveram mais falência orgânica $(54 \%$ versus $46 \%, p=0,004)$ e apresentaram maior necessidade de prótese respiratória por qualquer razão ( $57 \%$ versus $43 \%, p=0,0003$ ) (Tabela 6 ).

\section{DISCUSSÃO}

A infecção e suas conseqüências são as maiores causas de morte em UTI gerais ${ }^{13}$. A prevenção é extremamente importante e exige um conhecimento aprofundado das taxas de infecção, tipo e natureza da infecção, ambiente de aquisição (comunitária, hospitalar, UTI) e padrão dos organismos causadores. O conhecimento dos fatores de risco para o desenvolvimento de infecção, principalmente quando adquirida na UTI, e sua relação com a mortalidade é essencial. Este estudo de prevalência de um dia é o maior realizado no Rio Grande do Sul e pode servir como base para um estudo maior nacional e eventualmente ajudar a definir estratégias para diminuir as taxas de infecção nas UTI.

O estudo mostra que no ano de 2003, aproximadamente $70 \%$ dos 174 pacientes nas 16 UTI participantes estavam infectados. Entre os infectados, 51 (29\%) adquiriram infecção na UTI. A taxa de infecção foi maior que a encontrada nos diferentes estudos de prevalência de infecção em UTI na Europa, Canadá ou Austrália1 ${ }^{1.5,7}$. Em estudos prévios realizados no México e Brasil as taxas de infectados foram maiores, mas ficaram abaixo de $60 \% \%^{8,10}$. Contudo, a proporção de pacientes que adquiriram infecção na UTI foi semelhante em quase todos os estudos.

A taxa de mortalidade associada à infecção na UTI foi de $45 \%$ para os 104 pacientes com seguimento. Esta taxa é mais elevada que a observada em outros estudos fora do Brasil ${ }^{5-7}$, mas semelhante a dados brasileiros ${ }^{10,11}$. O estudo SEPSE BRASIL que encontrou uma mortalidade global de $46,6 \%$ e na região sul de $52,4 \%$ para sepse em UTI apresenta dados em concordância com os nossos $^{11}$. Não fica clara a razão para esta diferença em relação à Europa ou os EUA. Especula-se que vários fatores difíceis de avaliar contribuíram para este achado. A totalidade das UTI participantes era de hospitais terciários, as hospitalizações eram na maioria de urgência médica, os pacientes na sua maioria tinham infecção nosocomial e mais comorbidades. Neste sentido, a gravidade medida pelo APACHE II deste estudo foi maior que no estudo EPIC ou no estudo mexicano ${ }^{1,8}$. Contudo, este estudo também confirma alguns dos conhecidos fatores de risco: gravidade da doença, duração de hospitalização e o uso de técnicas invasivas (neste caso prótese em via aérea e cateteres). Entre os fatores de risco independentemente associados a óbito encontramos a idade, o escore APACHE II, a disfunção orgânica e a necessidade de prótese na via aérea por qualquer razão. Apesar de pneumonia não ter sido relacionada como um fator independente, o fator 'via aérea' indica que este deva ter influenciado o desfecho destes pacientes.

Outras comparações com o estudo EPIC são inevitáveis ${ }^{1}$. A prevalência de infecções adquiridas na comunidade foi mais elevada no nosso estudo ( $26 \%$ versus $13,7 \%$ ) em comparação com o Europeu. Entretanto, as 
taxas de prevalência observadas nos estudo mexicano $(23,9 \%)$ e um brasileiro $(20,8 \%)$ são comparáveis ${ }^{8,11}$. Questionários deste tipo são limitados e os resultados devem ser interpretados com cuidado. Esta avaliação "fotográfica" de um dia é limitada em tempo quando comparada com estudos de incidência e podem subestimar o problema. A identificação e a participação voluntária das UTI participantes podem gerar vieses de seleção. Certamente esta limitação está presente neste estudo, pois apenas 16 UTI participaram deste estudo, a maioria de Porto Alegre e com características acadêmicas. O potencial para o uso de métodos invasivos de monitorização e diagnóstico é supostamente maior neste tipo de UTI. Por fim, o preenchimento do questionário não foi supervisionado e a qualidade da informação não pode ser verificada (ex. o uso correto das definições de pneumonia).

Soma-se a estas limitações inerentes ao questionário, o diagnóstico preciso de infecção é difícil em pacientes de UTI. As culturas foram positivas em $52 \%$ dos pacientes com o diagnóstico de infecção na UTI. Muitas culturas consideradas causadoras de infecção podem ser resultados de colonização, um problema inerente a pacientes criticamente enfermos. Contudo, os isolados encontrados neste estudo, com predominância de cocos Gram-positivos, particularmente $S$ aureus, e organismos Gram-negativos, especialmente $P$ aeruginosa, estão de acordo com outros estudos realizados no Brasil e no exterior $8,9,14$.

Este estudo confirma a importância e o tamanho do problema da infecção nosocomial, particularmente a pneumonia e o trato respiratório inferior, resultado este muito semelhante aos diversos estudos com o mesmo desenho ${ }^{1,9,11}$. O estudo mostra também que a prevalência de infecção adquirida na UTI varia muito entre as diferentes UTI. Não foi possível, entretanto identificar um padrão de UTI com prevalência alta ou baixa. Os leitos de UTI geral do Hospital A apresentaram uma prevalência de $14 \%$ enquanto a UTI do Hospital B teve uma prevalência de $40 \%$. A UTI de trauma do Hospital K apresentou uma prevalência de $29 \%$ e a UTI de trauma do Hospital D teve uma prevalência de 59\%. Não fica clara a explicação para estas diferenças. Provavelmente, tanto as práticas no cuidado intensivo e a seleção dos pacientes estão implicadas nestas diferenças. Por exemplo, no Hospital B o déficit de leitos de UTI em relação ao número de leitos hospitalares e ao tamanho de sua emergência é maior que no Hospital A. Este fator pode levar a UTI do Hospital B a internar os pacientes mais graves e em piores condições. A UTI do Hospital D, referência para trauma, recebe também pacientes de outros tipos de emergência e pós-operatório de neurocirurgia.

O estudo confirma outros estudos em relação ao crescimento das infecções com microorganismo gram-positivo $^{14,15}$. Observa-se também que a maioria $(64 \%)$ dos isolados de $S$ Aureus era resistente a oxacilina. A identificação de isolados de $P$ Aeruginosa mostra a crescente preocupação com a resistência a antibióticos. A taxa de infecção por fungos, particularmente cândida, foi menor que a encontrada em outros estudos ${ }^{1,11}$.

Este tipo de estudo tem importância para o conhecimento da população infectada nas UTI. Esperamos que este estudo, sem similar na região Sul do Brasil, sirva como referência epidemiológica para ajudar a programar políticas de controle de infecção e represente um começo para futuras avaliações do impacto destas políticas no cuidado dos pacientes criticamente enfermos.

\section{AGRADECIMENTOS}

Agradecemos ao Dr. Márcio Soares (INCA) pela revisão do manuscrito

\section{REFERÊNCIAS}

01. Vincent JL, Bihari DJ, Suter PM et al - The prevalence of nosocomial infection in intensive care units in Europe. Results of the European Prevalence of Infection in Intensive Care (EPIC) Study. EPIC International Advisory Committee. JAMA, 1995;274:639-644.

02. Burke JP - Infection control - a problem for patient safety. N Engl J Med, 2003;348:651-656.

03. Angus DC, Linde-Zwirble WT, Lidecker $\mathrm{J}$ et al - Epidemiology of severe sepsis in the United States: analysis of incidence, outcome and associated costs of care. Crit Care Med, 2001;29:1303-1310.

04. Alberti C, Brun-Buisson C, Burchardi $\mathrm{H}$ et al - Epidemiology of sepsis and infection in ICU patients from an international multicentre cohort study. Intensive Care Med, 2002;28:108-121.

05. The outcome of patients with sepsis and septic shock presenting to emergency departments in Australia and New Zealand. Crit Care Resusc, 2007;9:8-18.

06. Karlsson S, Varpula M, Ruokonen E et al - Incidence, treatment, and outcome of severe sepsis in ICU-treated adults in Finland: the Finnsepsis study. Intensive Care Med, 2007;33:435-443.

07. Engel C, Brunkhorst FM, Bone HG et al - Epidemiology of sepsis in Germany: results from a national prospective multicenter study. Intensive Care Med, 2007;33:606-618.

08. Ponce de Leon-Rosales SP, Molinar-Ramos F, Dominguez-Cherit G et al - Prevalence of infections in intensive care units in Mexico: a multicenter study. Crit Care Med, 2000;28:1316-1321.

09. Toufen Junior C, Hovnanian ALD, Franca SA et al - Prevalence rates of infection in intensive care units of a tertiary teaching hospital. Rev Hosp Clin Fac Med Sao Paulo, 2003;58:254-259.

10. Silva E, Pedro MA, Sogayar AC et al - Brazilian Sepsis Epidemiological Study (BASES study). Crit Care, 2004;8:R251-R260.

11. Sales Junior JAL, David CM, Hatum R et al - Sepse Brasil: Estudo epidemiológico da sepse em unidades de terapia intensiva brasileiras. Rev Bras Ter Intens, 2007;18:9-17.

12. Knaus WA, Draper EA, Wagner DP et al - APACHE II: a severity of disease classification system. Crit Care Med, 1985;13:818-829.

13. Russell JA - Management of sepsis. N Engl J Med, 2006;355:1699-1713.

14. Vincent JL - Microbial resistance: lessons from the EPIC study. European Prevalence of Infection. Intensive Care Med, 2000;26:(Suppl1):S3-S8.

15. Friedman G, Silva E, Vincent JL - Has the mortality of septic shock changed with time. Crit Care Med, 1998;26:2078-2086. 